\title{
Diacronie
}

Studi di Storia Contemporanea

$N^{\circ} 14,2 \mid 2013$

Processo penale, politica, opinione pubblica (secoli XVIII-XX)

\section{Il caso di Ethel e Julius Rosenberg, la coppia che scelse di resistere}

\section{Cecilia Tognon}

\section{(2) OpenEdition \\ Journals}

\section{Edizione digitale}

URL: http://journals.openedition.org/diacronie/234

DOI: $10.4000 /$ diacronie.234

ISSN: 2038-0925

\section{Editore}

Association culturelle Diacronie

Notizia bibliografica digitale

Cecilia Tognon, « II caso di Ethel e Julius Rosenberg, la coppia che scelse di resistere », Diacronie

[Online], N 14, 2 | 2013, documento 9, Messo online il 01 août 2013, consultato il 19 avril 2019. URL : http://journals.openedition.org/diacronie/234 ; DOI : 10.4000/diacronie.234 


\title{
Diacronie
}

\section{Il caso di Ethel e Julius Rosenberg, la coppia che scelse di resistere}

\author{
Cecilia TOGNON*
}

Negli anni Cinquanta il processo per cospirazione intentato dal governo degli Stati Uniti a carico di Ethel e Julius Rosenberg si trasformò in uno dei casi giudiziari più famosi del secolo, valicando le aule di tribunale. Obiettivo di questo lavoro non è quello di produrre l'ennesimo verdetto di assoluzione o di colpevolezza, ma quello di ricostruire una vicenda tuttora affascinante, il cui interesse deriva in larga misura dalle figure dei protagonisti e dalla loro irremovibile scelta di resistere. I motivi del clamore suscitato dal loro caso giudiziario vengono ricercati nelle diverse fasi del processo, nei successivi rivolgimenti, nelle posizioni dell'accusa e della difesa e infine nelle pressioni esercitate dalle diverse forze coinvolte.

\section{Introduzione}

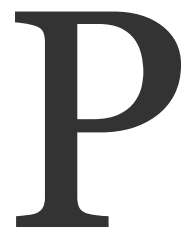

roprio perché ancora non conosce una conclusione univoca, la vicenda dei coniugi Rosenberg può essere raccontata cominciando da diversi punti di partenza. Nel narrarla si può per esempio iniziare dal 19 giugno 1953, giorno

dell'esecuzione della sentenza di morte per Julius ed Ethel Rosenberg, oppure dal 23 settembre 1949, quando il presidente degli Stati Uniti Truman annunciò alla nazione e al mondo che l'Unione Sovietica aveva fatto esplodere un'arma atomica il mese precedente; oppure dal 4 luglio 1966, quando negli Stati Uniti venne emanato il Freedom of Information Act (FOIA), legge sulla libertà di informazione grazie alla quale, non senza fatiche e resistenze, furono rese note migliaia di pagine di documenti governativi prima segreti che hanno permesso di chiarire alcuni punti oscuri e di illuminare verità per anni taciute. 
In questa ricostruzione del caso Rosenberg si adotta la cronologia forse più scontata, ossia si sceglie di cominciare con la data di inizio del processo a loro carico: il 6 marzo 1951. Questo perché, ad una prima parte dedicata ad una rapida descrizione del periodo in cui si celebrò il processo e degli imputati, seguirà una ricostruzione del processo stesso e della versione dei fatti che da questo emerse per evidenziare, infine, senza entrare nel merito punto per punto delle testimonianze, delle prove raccolte o dei numerosi documenti giudiziari, le particolarità del processo e dei capi d'accusa mossi. Ricostruire la vicenda di questa, suo malgrado, famosa coppia newyorchese non significa produrre l'ennesima sentenza di assoluzione o di condanna, ma semplicemente riconoscere che fu un caso emblematico di un'era, che appassionò, scosse e divise le coscienze di molti americani e non solo. Ciò che fu messo in scena fu davvero un grande spettacolo con finalità dichiarate e non che aveva almeno parte della sceneggiatura già scritta.

\section{Gli Stati Uniti degli anni Cinquanta}

Gli anni Cinquanta negli Stati Uniti non furono certo, come è noto, anni sereni, ma, al contrario, un periodo durante il quale molte libertà civili furono duramente messe alla prova. In quegli anni vigeva una legislazione severa che annoverava provvedimenti quali lo Smith Act «che perseguiva penalmente l'adesione a qualsiasi gruppo che sostenesse "il rovesciamento del governo con la forza e la violenza"»1. Nel 1947 il presidente Truman emanò «il decreto esecutivo 9835, che dava avvio a un programma di indagini per individuare qualsiasi «infiltrazione di persone inaffidabili» nell'apparato statale americano. Nei cinque anni successivi si indagò su sei milioni di dipendenti pubblici, e circa cinquecento furono licenziati per «lealtà discutibile» ${ }^{2}$; questo decreto, inoltre,

chiedeva al dipartimento di Giustizia di redigere un elenco delle organizzazioni a suo giudizio «totalitarie, fasciste, comuniste o sovversive [...] oppure miranti a modificare la forma di governo degli Stati Uniti con mezzi incostituzionali». Nel giudicare la fedeltà di un individuo alla nazione si sarebbe tenuto conto non solo

\footnotetext{
${ }^{1}$ ZINN, Howard, Storia del popolo Americano. Dal 1942 a oggi, Milano, il Saggiatore, 2010, p. 291.

2 Ibidem, p. 298. 
dell'iscrizione vera e propria, ma anche di un rapporto da simpatizzante con qualsiasi organizzazione compresa nella lista del segretario alla Giustizia3.

Sempre nel 1947 fu emanato il National Security Act che «istituì il National Security Council (NSC), un organo consultivo presidenziale con il compito di elaborare strategie integrate di politica estera, interna e militare; [...] istituì inoltre la Central Intelligence Agency (CIA) per coordinare le attività di spionaggio all'estero»4 .

L'Internal Security Act del 1950, invece, prevedeva la registrazione delle organizzazioni comuniste e l'instaurazione di un Comitato per il Controllo delle Attività Sovversive al fine di indagare persone ritenute sospette, a questa legge fu aggiunta una proposta dei

senatori liberal Hubert Humphrey e Herbert Lehman [che] proposero di creare centri di detenzione (in sostanza campi di concentramento) per i sospetti sovversivi, i quali, nel momento in cui il presidente avesse dichiarato uno stato di "emergenza per la sicurezza interna", sarebbero stati internati senza processo5.

Tutti questi provvedimenti, uniti alla parabola politica del senatore Joseph McCarthy e ad un'azione di massiccia propaganda interna che passava anche attraverso alcuni film hollywoodiani (mondo, quello del cinema, che subì esso stesso pesanti indagini ed epurazioni, con attori, registi e sceneggiatori finiti sulle liste nere se non addirittura in carcere, da parte del Comitato della Camera per le attività un-American, lo HUAC) contribuirono a creare un clima di paura e di isteria collettiva nei confronti del comunismo. Senza questo clima di Red Scare, simile a quello del primo dopoguerra, la vicenda dei Rosenberg sarebbe apparsa a tutti e fin dagli inizi come una sproporzionata parodia. Ed è solo nella cornice di questi provvedimenti legislativi, i quali non solo chiedevano una totale fedeltà alla propria nazione, ma rendevano anche possibili azioni giudiziarie sulla base delle simpatie politiche di ciascuno, che si può comprendere come sia stato possibile condannare due persone alla sedia elettrica per il reato di cospirazione (conspiracy).

Questo clima interno non veniva di certo favorito da ciò che accadeva fuori dai confini statunitensi. Nel gennaio del 1949 le forze comuniste cinesi entrarono a Pechino e nell'agosto dello stesso anno l'Unione Sovietica fece esplodere il suo primo ordigno atomico. Come se non bastasse nel giugno dell'anno successivo le truppe della Corea

\footnotetext{
3 Ibidem, p. 300.

4 TESTI, Arnaldo, Il secolo degli Stati Uniti, Bologna, Il Mulino, 2008, pp. 180-181.

5 ZINN, Howard, op. cit., p. 300.
} 
del Nord, paese incluso nella sfera di influenza sovietica, attraversarono il $38^{\circ}$ parallelo e invasero la Corea del Sud, protetta dagli Stati Uniti. Era l'inizio della guerra di Corea, che mobilitò l'opinione pubblica, anche progressista, e che contribuì a mantenere un clima di militarizzazione, sia in politica estera, sia interna.

\title{
2. Gli imputati, la difesa e l'accusa
}

Julius Rosenberg era nato nel 1918 a New York, suo padre era un ebreo emigrato dalla Russia zarista negli Stati Uniti e approdato, come molti altri, nel Lower East Side.

A sedici anni prendeva ancora sul serio la religione, ma a diciotto era già diventato un marxista. Nel febbraio del 1939 si laureò al City College di New York in ingegneria elettrica e sposò Ethel Greenglass poco dopo. Lavorò per il reparto comunicazioni dell'esercito fino al 1945, quando fu licenziato dopo che un'indagine rivelò che per ottenere il lavoro aveva giurato il falso, dichiarando di non appartenere al Partito Comunista. Lavorò poi per Radio Emerson, anche qui fino al licenziamento. Nell'ultimo periodo aveva aperto un'officina meccanica, assumendo suo cognato, David Greenglass. Nel 1950, al momento del suo arresto, l'officina era sulla via del fallimento ${ }^{6}$.

Ethel, anche lei ebrea e figlia di un immigrato bielorusso arrivato nel Lower East Side di Manhattan, era nata nel 1915

\begin{abstract}
una studentessa modello, diplomata prima del suo sedicesimo compleanno, con una voce straordinaria grazie alla quale fu scelta per cantare l'inno nazionale ai raduni scolastici. [...] Recitare e cantare erano le sue passioni. Faceva parte del Clark Street Players, un gruppo di teatro amatoriale, e dopo il diploma trovò impiego come segretaria. Nel 1935 era diventata un'attiva organizzatrice sindacale e lasciò il Clark Street Players per entrare nel più politicizzato gruppo teatrale Lavanburg Players7.
\end{abstract}

I due si incontrarono in una riunione del sindacato nel 1936, si sposarono nel 1939, il primo figlio, Michael, nacque nel 1943 e il secondo, Robert, nel 1947. Come le famiglie di appartenenza anche i Rosenberg conducevano una vita modesta.

\footnotetext{
${ }^{6}$ MEEROPOL, Robert, Quando il governo decise di assassinare mio padre e mia madre. Il figlio di Ethel e Julius Rosenberg racconta, Frankfurt am Main, Zambon, 2007, p. 24.

7 Ibidem, pp. 24-25.
} 
Nel 1950, al momento dell'arresto, essi non indicarono un legale di fiducia, pertanto fu il tribunale federale che assegnò loro due avvocati di ufficio, padre e figlio: Alexander Bloch difese Ethel Rosenberg e ad Emanuel "Manny” E. Bloch spettò la difesa di Julius Rosenberg.

Durante il processo la pubblica accusa fu assegnata al procuratore capo Irving $\mathrm{H}$. Saypol che, insieme ai suoi assistenti, rappresentò il governo degli Stati Uniti.

Il giudice del processo fu l'Onorevole Irving R. Kaufman, giudice federale a vita nominato dal presidente Truman.

Come gli imputati Rosenberg e Greenglass anche i Bloch, avvocati della difesa, erano ebrei, come lo era, peraltro, la pubblica accusa, rappresentata da Saypol e dal suo assistente Roy M. Cohn, e come lo era, infine, anche il giudice Kaufman. Ciò aiutò quelle forze che cercavano di allontanare le accuse di antisemitismo evocate, le quali non solo indebolivano il processo, ma facevano anche nascere tra l'opinione pubblica il sospetto che gli imputati potessero essere vittime di un qualche pregiudizio.

\section{Il processo}

Erano le 10.30 di martedì 6 marzo 1951, quando nel tribunale federale di New York City venne discusso il caso «United States of America vs. Julius Rosenberg, Ethel Rosenberg, Anatoli A. Yakovlev, also known as "John”, David Greenglass and Morton Sobell» ${ }^{8}$. Il capo di accusa era quello di cospirazione finalizzata allo spionaggio (conspiracy to commit espionage). La legge in base alla quale essi furono rinviati a giudizio era l'Espionage Act del 1917 e più precisamente il Titolo 50 sez. 32 (a) e 34 del codice degli Stati Uniti. L'Espionage Act subì numerosi emendamenti nel corso del tempo, ma trova ancora posto nel codice statunitense al Titolo 18 sez. 792 e seguenti. Questo fu il capo di imputazione letto al processo:

Whoever with intent or reason to believe that it is to be used to the injury of the United States or to the advantage of a foreign nation communicates, delivers or transmits or attempts to or aids or induces another to communicate, deliver or transmit to any foreign government or to any faction or party or military or Naval forces within a foreign country whether recognized or unrecognized by the United States, or to any representative, officer, agent, employee, subject or citizen thereof either directly or

\footnotetext{
${ }^{8}$ Rosenberg Trial, p. 1, URL:

<http://www.law.umkc.edu/faculty/projects/FTrials/Rosenberg/RosenbergTrial.pdf >, [consultato il 4 giugno 2013].
} 
indirectly any document, writing, code book, signal book, sketch, photograph, photograph negative, blueprint, plan, map, model note, instrument appliance or information relating to the National Defense shall be punished»9. «If two or more persons conspire to violate the provisions of Section 32 or 33 of this title and one or more of such persons does any act to effect the object of the conspiracy, each of the parties to such conspiracy shall be punished ${ }^{10}$.

La pena massima prevista era di venti anni di prigione, ma con questa eccezione: «Whoever violates this law in time of war shall be punished by death or by imprisonment for not more than thirty years» ${ }^{11}$.

Julius Rosenberg era stato arrestato il 17 luglio del 1950; a sua moglie Ethel toccò la stessa sorte meno di un mese dopo, l'11 agosto. La giuria, composta da undici uomini e una donna, si ritirò per decidere il 28 marzo; il giorno successivo emanò un verdetto di colpevolezza. Il 5 aprile 1950 il giudice Irving Kaufman condannò Morton Sobell a trent'anni di prigione e i Rosenberg alla pena di morte.

Prima di fornire il quadro degli eventi così come emerse nel processo è il caso, per inquadrare meglio alcuni fatti, circostanze e personaggi, di risalire velocemente la catena di confessioni e delazioni più o meno genuine che portò gli agenti dell'FBI a bussare al modesto appartamento nel Knickerbocker Village per arrestare Julius Rosenberg.

Nel gennaio del 1950 il fisico tedesco Klaus Fuchs, fuggito in Inghilterra dopo la presa di potere da parte dei nazisti, confessò di aver fornito all'Unione Sovietica importante materiale sulla costruzione della bomba atomica quando faceva parte, come fisico, della delegazione inglese che lavorava al Progetto Manhattan, programma segreto avviato durante la Seconda guerra mondiale e che condusse gli Stati Uniti allo sviluppo dell'arma atomica. Il suo corriere era Harry Gold, un chimico di Philadelphia che passava le informazioni ottenute dal fisico ad un agente sovietico: Anatoli Yakovlev. A sua volta l'arresto di Harry Gold e la sua confessione portarono a David Greenglass, che, interrogato dall'FBI, confessò di aver passato informazioni sull'ordigno atomico all'Unione Sovietica e coinvolse in questo terribile reato anche sua sorella Ethel e il marito di lei, Julius. ${ }^{12}$

\footnotetext{
9 Ibidem, p. 33.

${ }^{10}$ Ibidem.

${ }_{11}$ SCHNEIR, Walter, SCHNEIR, Miriam, Invitation to an Inquest. A New Look at the Rosenberg-Sobell Case, New York, Doubleday \& Company, 1965, p. 168.

${ }^{12}$ SCHRECKER, Ellen, The Age of McCarthyism: A Brief History with Documents, Boston-New York, Bedford-St. Martin's, 2001, pp. 38-40.
} 
A questo punto appare chiaro come tra i diversi testimoni del processo i tre personaggi chiave portati alla sbarra dall'accusa fossero: Harry Gold, David Greenglass e Ruth Greenglass. Lo schema degli eventi che si costruì dalle loro testimonianze è il seguente. Nell'agosto del 1944 David Greenglass entrò a lavorare come meccanico all'interno dei laboratori di Los Alamos, dove si stava sviluppando la bomba atomica. Alla fine di novembre, David imparò da sua moglie Ruth la natura degli esperimenti che si tenevano a Los Alamos: a dire di Ruth, Julius l'aveva informata di ciò. In diverse occasioni nei mesi successivi David passò a Julius alcune informazioni, tra cui degli schizzi di lenti metalliche usate come detonatori dell'ordigno, un elenco di scienziati che lavoravano a Los Alamos e i nomi di alcune possibile reclute. L'evento cruciale ai fini dell'accusa fu quello che unisce Harry Gold, David Greenglass e Julius Rosenberg. Disse Saypol nell'arringa finale «Harry Gold, who furnished the absolute corroboration of the testimony of the Greenglasses, forged the necessary link in the chain that points indisputably to the guilt of the Rosenbergs ${ }^{13}$. Infatti Gold, oltre a fornire la conferma a quanto affermato dai Greenglass, costituiva quel legame necessario tra le informazioni sulla bomba atomica, Julius Rosenberg e l'agente sovietico Yakovlev: senza quanto affermato da Gold, semplicemente non ci sarebbe stato nessun caso di cospirazione. L'incontro in questione avvenne la prima domenica di giugno del 1945, al 209 di North High Street in Albuquerque, quando un uomo, allora sconosciuto ai Greenglass, bussò al loro appartamento presentandosi con la frase che tanto peso avrà per le sorti dell'imputato coinvolto: «Julius sent me»14. David al momento della visita non aveva il materiale pronto, che poté consegnare al corriere (ricevendo in cambio cinquecento dollari), solo nel pomeriggio. Un altro momento cruciale nella ricostruzione dei testimoni si colloca nel settembre del 1945 a New York quando David disse a Julius «I think I have... a pretty good description of the atom bomb»15. È infatti in questa occasione che avvenne il coinvolgimento di Ethel Rosenberg. David aveva preparato l'abbozzo di una bomba a implosione con inerente descrizione e fu nel salotto dei Rosenberg, a dire di David, che «Ethel did the typing and Ruth and Julius and Ethel did the correction of the grammar» ${ }^{16}$. Infine, nel febbraio del 1950, poco dopo l'arresto di Fuchs, Julius fece visita a David invitandolo a lasciare il paese, ripetendo tale consiglio prima a metà aprile e poi in giugno, quando Julius vide David per l'ultima volta.

Il primo testimone della difesa chiamato a rispondere di tali accuse, fu Julius che, interrogato dal suo avvocato Emanuel Bloch, negò completamente qualsiasi attività di

${ }^{13}$ SCHNEIR, Walter, SCHNEIR, Miriam, op.cit., p. 155.

${ }^{14}$ Ibidem, p. 125.

15 Ibidem, p. 126.

${ }^{16}$ Ibidem, p. 127. 
spionaggio rispondendo a molte domande con le stesse tre parole: «I did not»17. Più volte il giudice Kaufman interruppe l'avvocato Bloch per interrogare l'imputato circa le sue idee politiche, ad alcune domande Julius si rifiutò di rispondere, affermando che non avrebbe risposto a domande su «membership in any political organization like Communist Party»18. Alla prima domanda delle pubblica accusa «concerned Julius's "associates... at City College" and "any common ... activities which brought this group ... together." Julius soon invoked the Fifth Amendment, saying he would not answer questions about the Young Communist League or Communist Party» ${ }^{19}$.

L'ultima testimone della difesa fu Ethel Greenglass Rosenberg che fu interrogata da Alexander Bloch. Anche lei come suo marito negò tutte le accuse sulle attività di spionaggio. Ethel inoltre testimoniò che lei non aveva mai battuto a macchina nulla di relativo alla difesa nazionale. Dopo l'arresto di Julius, Ethel era stata portata due volte (il 7 e l'11 agosto) davanti a un Gran Giurì federale. Entrambe le volte aveva invocato di frequente il suo diritto, costituzionalmente garantito, contro l'auto-incriminazione. Ciò fu sfruttato da Saypol durante il processo: egli dedicò ben metà del suo contro interrogatorio ad Ethel a sottolineare questo comportamento della imputata. Anche il giudice Kaufman non mancò l'occasione di sottolineare il comportamento di Ethel Rosenberg: «said the jury could consider the matter in evaluating the truthfulness and credibility of Mrs. Rosenberg» ${ }^{20}$.

Questo e molto altro è ciò che venne fuori durante i quattordici giorni del processo durante i quali si alternarono al banco dei testimoni altre persone, furono analizzati i reperti e l'accusa e la difesa si soffermarono su molti altri dettagli che qui non verranno presi in considerazione.

La mattina del 29 marzo la giuria emanò infine il suo verdetto: «We the Jury find Julius Rosemberg guilty as charged. We the Jury find Ethel Rosenberg guilty as charged. We the jury find Morton Sobell guilty as charged»21. Ringraziandoli il giudice Kaufman disse loro «My own opinion is that your verdict is a correct verdict [...] I can't find words to describe this loathsome offense» ${ }^{22}$. La sentenza del giudice Kaufman sull'entità della pena da infliggere fu fissata ad una settimana dopo.

Egli si espresse il 5 aprile con un giudizio destinato a rimanere famoso: fama che deriva dalla gravità delle parole pronunciate, che restituiscono tutte le paure e le

\footnotetext{
17 Ibidem, p. 143.

18 Ibidem, p. 144.

19 Ibidem, p. 147.

${ }^{20}$ Ibidem, p. 150.

${ }^{21}$ Ibidem, p. 167.

22 Ibidem.
} 
angosce di un'epoca e che portano all'interno di un'aula di tribunale una guerra lontana, ma molto vicina all'opinione pubblica statunitense; parole, infine, che suggeriscono responsabilità che nella loro gravità appaiono sproporzionatamente attribuibili a quella coppia di newyorchesi:

I consider your crime worse than a murder [...] I believe your conduct in putting into the hands of the Russians the A-Bomb years before our best scientists predicted Russia would perfect the bomb has already caused, in my opinion, the Communist aggression in Korea, with the resultant casualties exceeding 50,000 and who knows but that millions more of innocent people may pay the price of your treason. Indeed, by your betrayal you undoubtedly have altered the course of history to the disadvantages of our country. No one can say that we do not live in a constant state of tension. We have evidence of your treachery all around us every day for the civilian defense activities throughout the nation are aimed at preparing us for an atom bomb attack ${ }^{23}$.

Aggiunse poi:

I... assume that the basic Marxist goal of world revolution and the destruction of capitalism was well known to the defendants, if in fact not subscribed to by them, when they passed what they knew was this nation's most deadly and closely guarded secret weapon to Soviet agents. [...] The evidence indicated quite clearly that Julius Rosenberg was the prime mover in this conspiracy. However, let no mistake be made about the role which his wife, Ethel Rosenberg, played in this conspiracy. [...] She was a full-fledged partner in this crime. [...] Love for their cause dominated their lives- it was even greater than their love for their children. [...] I am convinced beyond any doubt of your guilt. [...] The sentence of the Court... is, for the crime for which you have been convicted, you are hereby sentenced to the punishment of death, and it is ordered... you shall be executed according to the law 24 .

A questa sentenza seguirono numerose istanze di appello, petizioni internazionali e manifestazioni, interventi di numerosi personaggi (tra gli altri: Pablo Picasso, il premio nobel per la chimica Harold C. Urey, Albert Einstein, Jean-Paul Sartre, la sorella di Bartolomeo Vanzetti e un appello orale di papa

${ }^{23}$ FERRARI, Giorgio, Ombre Rosse: il caso Rosenberg e la Guerra Fredda, Milano, BookTime, 2010 , p. 102.

${ }^{24}$ SCHNEIR, Walter, SCHNEIR, Miriam, op.cit., pp. 170-171. 
Pio XII affinché fosse commutata la pena dei Rosenberg), sospensioni e rinvii dell'esecuzione, comprese due domande di grazia ai due Presidenti, prima a Truman e poi al suo successore Eisenhower. In seguito ai numerosi respingimenti, un'ultima speranza sembrò arrivare il 17 giugno del 1953 quando William O. Douglas, un giudice della Corte Suprema, sospese l'esecuzione per poter analizzare meglio una discrepanza fra due leggi: l'Espionage Act del 1917 e la legge sull'energia atomica del 1946. Di gran fretta venne allora nuovamente convocata in seduta straordinaria la Corte Suprema, che il 19 giugno 1953 annullò il rinvio concesso dal giudice Douglas; la sera di quello stesso giorno, poco dopo le otto, prima Julius e poi Ethel Rosenberg trovarono la morte che li aspettava su una sedia elettrica nel penitenziario federale di Sing Sing.

\section{Rivelazioni successive}

La vicenda dei coniugi Rosenberg non si spense in quella sera del giugno 1953: l'accanimento e la sproporzione di quella condanna erano elementi difficilmente trascurabili. Inoltre i Rosenberg non avevano lasciato dietro di loro solo un'eredità morale ed ideologica, ma anche un'altra ben più concreta: i loro due figli Michael e Robert la cui custodia legale era stata affidata dai due genitori al loro avvocato Manny Bloch. Michael e Robert furono poi adottati dai coniugi Meeropol di cui assunsero il cognome. Anche i Meeropol erano stati membri del Partito Comunista, appartenevano dunque alla stessa comunità politica dei Rosenberg, i quali preferirono far crescere i loro figli da militanti come loro piuttosto che dai parenti e Michael e Robert diventarono in qualche modo i figli del movimento. Fu forse anche per questo che essi decisero negli anni Settanta di esporsi e di intraprendere una serie di iniziative legali volte ad ottenere il rilascio di molti documenti fino a quel momento mantenuti segreti e la riapertura del caso. Se il secondo obiettivo non riuscì, essi ottennero importanti vittorie sul primo fronte. Nel luglio del 1975 presentarono «una corposa denuncia ai sensi del FOIA [legge sulla libertà di informazione] contro sette agenzie governative» 25 . Nell'autunno dello stesso anno l'FBI accordò il suo primo rilascio di documenti con parecchie centinaia di pagine. Molto fu ciò che emerse da questi e da altri documenti successivamente rilasciati (un totale di trecentomila pagine), da cui emerse che la condotta del giudice Irving Kaufman, che aveva discusso con gli avvocati dell'accusa in

25 MEEROPOL, Robert, op. cit., p. 195. 
merito alla possibilità di infliggere la pena capitale; «altri documenti rivelavano che Kaufman aveva usato il Dipartimento di giustizia e l'FBI per intercedere presso la Corte di Appello e la Corte Suprema affinché accelerassero i giudizi di appello e assicurassero così una più rapida esecuzione» ${ }^{26}$. Un altro documento rivelava che, mentre il giudice Douglas stava riflettendo circa la possibilità della sospensione dell'esecuzione, la sera del 16 giugno 1953,

il procuratore generale Herbert Brownell si era incontrato segretamente con il giudice capo della Corte Suprema Fred Vinson. Il documento concludeva che, durante quell'incontro, il giudice capo Vinson "disse che se veniva accordata una sospensione, il giovedì mattina avrebbe riunito l'intera Corte in sessione straordinaria per renderla vana" [...]. Esso costituisce la prova documentale che il procuratore generale, uno dei massimi funzionari esecutivi del nostro paese, aveva cospirato con il capo dei giudici degli Stati Uniti per annullare la sospensione di un'esecuzione, stabilita da un altro giudice che sedeva nella Corte Suprema, prima di aver letto le basi legali su cui tale sospensione era stata accordata, dal momento che essa non venne scritta fino al mattino successivo ${ }^{27}$.

Altri documenti riferivano di un incontro tra Harry Gold e David Greenglass organizzato dai legali dell'accusa e avvenuto più di due mesi prima dell'inizio del processo, durante il quale i due testimoni si accodarono circa quella frase in codice che, secondo le ricostruzioni processuali, venne utilizzata da Gold per presentarsi a Greenglass in quella mattina del giugno 1945: «Vengo da parte di Julius», frase che, come già ricordato, rappresenta l'anello fondamentale, l'evento per il quale Julius Rosenberg venne condannato.

Nel 1995 fu scoperta un'ulteriore mole di materiale, precedentemente tenuto sotto la massima segretezza, che conteneva anche informazioni sui Rosenberg, ossia la trascrizione del progetto Venona. Con questo nome si identifica una collaborazione avvenuta tra le agenzie di spionaggio statunitensi e quelle inglesi volta a intercettare e a decifrare i messaggi in codice che il KGB inviava dal consolato sovietico di New York a Mosca, soprattutto durante gli anni della Seconda guerra mondiale. Le informazioni contenute in questi documenti non furono utilizzate durante il processo a carico dei Rosenberg, poiché ritenute potenzialmente pericolose per la sicurezza nazionale.

Anche queste rivelazioni come quelle precedenti non permettono di chiarire completamente la vicenda e di far luce sulla totalità dei fatti. Per i sostenitori della

${ }^{26}$ Ibidem, p. 199

${ }_{27}$ Ibidem, p. 200. 
completa innocenza dei Rosenberg le trascrizioni di Venona sono ben lungi dall'essere una fonte attendibile e indipendente, tuttavia, come sottolinea il figlio stesso dei Rosenberg, Robert, sostenere che il governo abbia potuto falsificare il contenuto di quelle trascrizioni è una cosa, ma ben altra è arrivare alla conclusione che ciò sia successo, senza poter addurre nessuna prova concreta.

Probabilmente sciogliere tutti i nodi di questo groviglio frutto di volontarie menzogne e di studiate strategie politiche, di disinformazione, di mezze verità, di bugie e di silenzi, figli più della paura che della malafede, non sarà mai possibile. A impedirlo è anche il tempo trascorso, gli interessi forti e divergenti che nel passato e ancora nel presente dividono le forze e gli attori coinvolti.

Ciò che non può essere negato è che, dietro quel processo, c'era una strategia, c'era la volontà di rappresentare gli imputati in un certo modo, c'era il desiderio non tanto di punire quei due sposi del Lower East Side, ma quello che essi rappresentavano. Il grado con cui quel processo sia stato alterato non è facilmente quantificabile, ma è certo che le irregolarità sono state numerose, il processo non fu condotto in maniera equa, il verdetto di colpevolezza affrettato e la condanna a morte sproporzionata, soprattutto se paragonata a quella del reo confesso David Greenglass, a cui spettarono quindici anni di reclusione.

Tuttavia, anche alla luce di quanto emerso dalle trascrizioni di Venona e da quanto dichiarato da Morton Sobell nel settembre del 2008 al «The New York Times», su qualcosa si è giunti ad una qualche convergenza: «nessuno dei due aveva commesso le azioni per le quali furono condannati; il governo degli Stati Uniti aveva sempre saputo che Ethel Rosenberg non era una spia di alcun genere» ${ }^{28}$. Quest'ultima posizione è stata confermata anche da David Greenglass che nel dicembre del 2001 ammise in un'intervista per il programma televisivo Sixty Minutes II di aver mentito sotto giuramento quando dichiarò di ricordare la sorella che batteva a macchina i suoi appunti. Sulla completa innocenza di Julius, invece, permangono dei dubbi in quanto la possibilità che egli abbia trasmesso informazioni di carattere industriale-militare ai sovietici non è esclusa, ma oltre a non essere del tutto appurata, essa non rappresenta, come sostengono i figli, il capo d'accusa per il quale loro padre fu condannato e ucciso.

\section{Sull'orlo della rottura}

${ }^{28}$ Ibidem, p. 291. 
Se ancora oggi ci troviamo di fronte ad una situazione complessa, quella che dovettero affrontare Manny Bloch e suo padre fu un compito ai limiti del possibile. A renderlo tale era l'accusa che pesava sulle spalle degli imputati, quella cioè di cospirazione finalizzata allo spionaggio.

\begin{abstract}
Prosecutors like conspiracy charges because the law in this country holds everyone involved in the conspiracy responsible for all the acts of any of the conspirators in furtherance of the conspiracy. And all the prosecutors need to show to prove that a conspiracy exists is that two or more people got together, made an illegal plan and took one overt act to move that scheme forward. It could be as simple as agreeing to make a phone call or arranging a meeting.

In order to prove a conspiracy the prosecution must demonstrate that the defendants joined together in a common plan'29.
\end{abstract}

Difendersi da questo tipo di accuse è compito arduo, poiché bisogna riuscire a dimostrare il proprio non coinvolgimento in qualcosa, il non essersi mai incontrati con qualcuno coinvolto in una cospirazione, il non aver organizzato un piano illegale e il non aver posto in essere nessun atto manifesto (compresa una semplice chiamata) al fine di realizzare tale piano. Il reato di cospirazione sussiste anche se non si consegue lo scopo desiderato, ciò che conta è l’intento.

Even the equivalent of a corpus delicti - to prove indisputably that a crime actually occurred - is lacking. [...] This legal truism is particularly meaningful in the Rosenberg-Sobell trial, where the primary acts charged against the defendants consisted of espionage conversations and transactions with friends and relatives whom they were seeing socially during the same period. If the defendants were innocent, what more - other than to deny the charges - might they have done to prove this? How, for example, could the Rosenbergs have offered an alibi when a principal locale of the alleged crime was their own living room and kitchen? The fact of the matter is that the accusation against the defendants were essentially irrefutable, offering them no avenue for definitive rebuttal 30 .

Nonostante la relativa facilità per l'accusa di giungere ad un verdetto di colpevolezza, anche grazie alle disponibilità dei mezzi e delle risorse del governo

29 ID., «6o Years Too Late/ 60 ans trop tard, La Revue des Ressource», 29 fevrier 2012, URL: <http://www.larevuedesressources.org/6o-years-too-late-6o-ans-trop-tard,2266.html>, [colsultato il 17 giugno 2013].

${ }^{30}$ SCHNEIR, Walter, SCHNEIR, Miriam, op. cit., pp. 157-158. 
degli Stati Uniti in confronto a quelle utilizzabili degli imputati, quello che era uno degli obiettivi principali del processo intentato, ossia la dimostrazione all'intera nazione e al mondo libero che la costruzione di un ordigno atomico da parte dell'Unione Sovietica e la conseguente perdita del primato statunitense non poteva essere spiegata altrimenti se non con un tradimento, non venne raggiunto. Tutto sfumò di fronte al silenzio ostinato, «consapevole e recalcitrante» 31 , semplice ma allo stesso tempo difficile e potentissimo che ha permesso ai Rosenberg di diventare «la coppia più tragica della guerra fredda»32. Sopra tutto, sopra i tradimenti del fratello e del cognato, sopra i titoli dei giornali, sopra le manifestazioni di massa e sopra la terribile possibilità di perdere i propri figli, si stagliano chiare e composte le figure di Ethel e Julius che rispondono ad ogni accusa negando il proprio coinvolgimento e proclamando la propria innocenza fino all'ultima lettera ai loro bambini 33 , fino all'ultimo istante, fino a quando Ethel, già morto il congiunto, risponde al rabbino andato a portare consolazione negli ultimi momenti di vita e a cercare una confessione che possa salvarla: «Non ho nomi da fare. Sono innocente. Sono pronta a morire»34. La possibilità di confessare, di fare dei nomi, di non interrompere la catena degli arresti, di rinnegare la propria appartenenza ideologica, era stata offerta più volte agli imputati, che in cambio avrebbero avuta salva la vita (quantomeno Ethel), ma il loro rifiuto netto, che li ha consegnati alla storia ha anche impostato la loro difesa, una difesa che non è riuscita a salvarli, ma che fu vincente per la causa che essi rappresentavano.

Secondo Jacques Vergès un processo penale può assumere lo stile di un processo di rottura o quello di connivenza, a determinarlo è l'atteggiamento

${ }^{31}$ MEEROPOL, Robert, Quando il governo decise di assassinare mio padre e mia madre, cit. p. 305 .

$3^{2}$ VERGÈS, Jacques M., Strategia del processo politico, Torino, Einaudi, 1969, p. 115.

33 «Carissimi bambini miei adorati, [...]Certo, all'inizio piangerete amaramente per noi, ma non piangerete da soli. Questa è la nostra consolazione e alla fine sarà anche la vostra. Un giorno anche voi dovrete convincervi che la vita merita di essere vissuta. Consolatevi perché ancora adesso, mentre la fine della nostra vita si avvicina lentamente, noi lo crediamo con una convinzione che sconfigge i nostri carnefici! La vita vi insegnerà anche che il bene non può fiorire in mezzo al male, che la libertà e tutto ciò che vale a rendere una vita veramente ricca $\mathrm{e}$ degna d'essere vissuta devono essere talvolta conquistati a caro prezzo. [...] Ricordate sempre che eravamo innocenti e che non abbiamo potuto far torto alla nostra coscienza. Vi stringiamo a noi e vi baciamo con tutta la nostra forza. Con amore, papà e mamma Julie e Ethel». MEEROPOL, Robert, MEEROPOL, Michael, Siamo vostri figli, Milano, Garzanti, 1976, pp. 284285 .

34 FERRARI, Giorgio, op. cit., p. 153. 
dell'accusato di fronte all'ordine pubblico, «se lo accetta, il processo è possibile e costituisce un dialogo tra l'accusato che spiega il proprio comportamento ed il giudice i cui valori vengono rispettati. Se invece lo rifiuta, l'apparato giudiziario si disintegra: siamo allora al processo di rottura»35. La difesa dei Rosenberg rientra all'apparenza nel processo di connivenza, in quanto c'è un'accettazione dell'apparato giudiziario, manifestata anche dale domande di grazia presentate al Presidente; tuttavia come afferma lo stesso Vergès ci troviamo di fronte ad un processo di «rottura aperta o di finta connivenza: l'antigiustizia. Qui, innocente è il colpevole, colpevole è l'innocente» 36 , infatti dall'inizio alla fine ciò che avrebbe salvato Julius ed Ethel non sarebbe stata una prova a favore della loro innocenza, ma una confessione della loro colpevolezza. In un celebre processo di rottura, quale fu quello di Dimitrov, l'imputato affermò con fierezza la propria appartenenza ideologica e imposta la propria difesa in maniera strategica privando l'accusa dell'iniziativa, nel processo ai Rosenberg in qualche modo avvenne il contrario. Probabilmente però essi non hanno voluto rendere politico il carattere del proprio processo poiché era esattamente ciò che avrebbe voluto il governo americano. Al banco degli imputati non sedevano solo Julius ed Ethel, ma anche tutti i comunisti americani e la più grande famiglia dei progressisti di sinistra. Nonostante la facciata apolitica del processo, costanti, fino al giudizio finale, furono i riferimenti all'appartenenza ideologica e militante degli imputati. È infatti alle domande sul Partito Comunista che essi si rifiutarono di rispondere, rifiutando di stare al gioco del governo, ma allo stesso tempo assecondandolo fino a rivolgerlo contro l'accusa stessa, «molti errori giudiziari non furono necessariamente in partenza errori politici. Lo diventarono solo col fallimento dell'impresa»37. Nel caso Rosenberg l'evoluzione verso un errore politico iniziò a farsi sempre più manifesta in seguito alla condanna a morte inflitta dal giudice Kaufman e ai successivi e ripetuti respingimenti delle domande di grazia, poiché ai primi comitati che denunciavano l'iniquità del processo, si aggiunsero proteste contro la sproporzione della pena inflitta e i movimenti contrari alla pena di morte, che potevano anche considerare imparziale lo svolgimento dell'azione penale. Alle proteste a livello nazionale si

35 VERGÈS, Jacques M., op. cit., p. 15 .

${ }^{36}$ Ibidem.

37 Ibidem. 
sommarono quelle provenienti da altri paesi, a cominciare dalla Francia in cui era più forte la componente che credeva che i Rosenberg fossero vittime dell'isteria generale anticomunista, ma anche in Inghilterra e in Italia si svilupparono movimenti di solidarietà, sia tra i comunisti, sia tra le forze moderate, maggiormente ispirate dal senso di umanità e di giusta proporzione della pena. Nei paesi del blocco sovietico il caso Rosenberg diventò pretesto e simbolo dell'oppressione capitalistica e, col passare del tempo, molti, anche tra i moderati americani, capirono che il processo sarebbe diventato un boomerang poiché in caso di esecuzione della condanna a morte i Rosenberg sarebbero assurti al ruolo di vittime del complotto americano anticomunista e in caso di clemenza si sarebbe lasciato spazio a ipotesi di debolezza del governo americano di fronte all'Unione Sovietica. Ecco dunque che la scelta, forse non del tutto strategica, ma anche morale, di non confessare e di resistere si rivelò vincente. I Rosenberg però appartenevano anche ad un'altra comunità, quella ebraica, la quale non gradì particolarmente il clamore che stava suscitando l’intera vicenda. L'American Jewish Committee (AJC) guardava con grande timore a quanto stava succedendo e mantenne una politica decisa a non soffiare ulteriormente sul fuoco e a prendere le distanze sia dal comunismo, sia dal The National Committee to Secure Justice in the Rosenberg Case e soprattutto da qualsiasi accusa di antisemitismo che veniva lanciata nei confronti del processo:

\footnotetext{
In recent months Communist have been attempting to agitate concerning these condemned persons and to inject into the situation a suggestion that there are antiSemitic implications to the conviction and the sentences. The AJC believes there is no foundation for such charges. Nevertheless, certain groups are persisting in an attempt to make this a public question on these grounds ${ }^{38}$.
}

Tuttavia, il veder montare la protesta diede forza ai Rosenberg che perseverarono nella loro difesa, anche psicologicamente impegnativa, fino alla fine, fino a poter condividere le parole pronunciate da Socrate dopo la sua

38 Political Philosophy/ Communism File, AJC Subject Files Collection. Material on the Rosenbergs, staff committee minutes, AJC work regarding Communism, April 1951. (6), p. 1, American Jewish Committee (AJC),

URL: < http://www.ajcarchives.org/ajcarchive/DigitalArchive.aspx > [consultato il 18 giugno 2013]. 
condanna, «e ora non mi pento di questa difesa. Anzi, vedete, preferisco la morte, dopo una simile difesa, assai più che la vita ottenuta in quell'altro modo» 39 .

Non si sta qui sostenendo la tesi della completa innocenza dei Rosenberg: quello che si vuole evidenziare è l'onda lunga che questo caso giudiziario ha lasciato dietro di sé, i personaggi che, anche inconsapevolmente, ha creato e la particolarità di un processo in cui si affrontavano il governo degli Stati Uniti e due personaggi certamente non potenti, la cui dialettica si è sprigionata in tutta la sua forza quando questi ultimi decisero di non cedere.

39 VERGÈS, Jacques M., op. cit., p. 53. 


\section{* L'autore}

Cecilia Tognon è iscritta al corso di Laurea Magistrale in Scienze storiche presso l'Università di Bologna.

URL: < http://www.studistorici.com/progett/autori/\#Tognon >

\section{Per citare questo articolo:}

TOGNON, Cecilia, «Ill caso di Ethel e Julius Rosenberg, la coppia che scelse di resistere», Diacronie. Studi di Storia Contemporanea : Processo penale, politica, opinione pubblica (secoli XVIII-XX), 29/08/2013,

URL:< http://www.studistorici.com/2013/08/29/tognon_numero_14/ >

Diacronie Studi di Storia Contemporanea $\widehat{\beta}$ www.diacronie.it

Risorsa digitale indipendente a carattere storiografico. Uscita trimestrale.

redazione.diacronie@hotmail.it

Comitato di redazione: Marco Abram - Jacopo Bassi - Luca Bufarale - Alessandro Cattunar - Elisa Grandi - Deborah Paci - Fausto Pietrancosta - Matteo Tomasoni - Luca Zuccolo

Diritti: gli articoli di Diacronie. Studi di Storia Contemporanea sono pubblicati sotto licenza Creative Commons 2.5. Possono essere riprodotti a patto di non modificarne i contenuti e di non usarli per fini commerciali. La citazione di estratti è comunque sempre autorizzata, nei limiti previsti dalla legge. 